\title{
Problems Connected with the TEM Determination of the Sizes of Small Defect Clusters
}

\author{
By K.-H. Katerbau \\ Max-Planck-Institut für Metallforschung, Institut für Physik, Stuttgart, Germany (BRD)
}

(Received 4 June 1974)

\begin{abstract}
In general, the contrast width and the visibility of small defect clusters observable by TEM is strongly dependent on the orientation and thickness of the foil examined, and on the depth positions of the defects in the foil. This dependence is not well known quantitatively. For example, the influence of the depth oscillations on the contrast of small clusters imaged under kinematical imaging conditions has not been studied in detail. Most of the contrast calculations to date have been performed for thick foils and for two-beam imaging conditions [Rühle, M. (1967). Phys. Stat. Sol. 19, 263, 279; Eyre, B. L. (1972). Proc. Intern. Discuss. Meeting Defects in Refractory Metals, Mol, 1971. Edited by R. de Batist, J. Nihoul \& L. Stals, p. 311 . Mol: SCK/CEN.] In practice, however, investigations of the size distribution of defect clusters are performed preferentially in fairly thin areas of the transmission foils. The reason is that, because of the effects of chromatic aberration, small defect clusters with sizes near the visibility limit are best observable in thin foils [cf., e.g., Noggle, T. S., Oen, O. S. \& Crump. I. I. I. (1970). 28th Ann. EMSA Meeting, Houston, Texas, p. 406.] Often these clusters are imaged under conditions where systematic higher-order reflexions have considerable intensity. In this paper many-beam calculations are reported for small clusters in foils of various thicknesses including thin foils. A time-saving method [Häusserman, F., Katerbau, K. -H., Rühle, M. \& Wilkens, M. (1973). J. Microsc. 98, 135] is used for the calculations in which the foil thickness and the depth position of the defect in the foil are eliminated from the integration procedures. In the $N$-beam case the differential equations of the Bloch-wave type [Wilkens, M. (1964). Phys. Stat. Sol. 6, 939] are integrated for $N$ different initial conditions for each column. From this $N$-fold set of solutions the contrast profiles can be easily calculated for any value of the foil thickness and the depth position and for $N$ different directions of incidence of the primary beam. This method has proved to be very useful for the calculation of the contrast of defects with localized strain fields such as small dislocation loops. The following results were obtained: (I) There is a strong quasiperiodic depth dependence of the contrast of small defect clusters not only under dynamical but also under kinematical imaging conditions. The periodicity is equal to the effective two-beam extinction length which belongs to the excitation error used. The contrast may change from a very high to a very low value depending on experimental parameters such as foil thickness, depth position of the loop inside the foil, excitation error and the effective divergence of the illuminating electron beam. (II) The ratio $R$ of the width of the contrast dot to the true size of the defect cluster is also dependent on these experimental parameters. $R$ may vary between $0 \cdot 9$ and $1 \cdot 6$. [The value $R=1 \cdot 0 \ldots 1 \cdot 2$ as occasionally used in the literature (Rühle, 1967; Eyre, 1972) is certainly too small in general.] Therefore, for a reliable evaluation of the size distribution, e.g. of small dislocation loops, a careful control of the experimental parameters is required. Further results of our calculations will be published by Katerbau, K.-H., Rühle, M. \& Wilkens, M. L(1974). Proc. 8th Intern. Congress on Electron Microscopy, Canberra].
\end{abstract}

